\title{
Effects of Surface Termination on Directional Emission from Photonic Crystal Waveguides
}

\author{
K. B. Chung* \\ School of Electronic and Electrical Engineering, Hongik University, Seoul 121-791, Korea
}

(Received February 26, 2008 : revised March 20, 2008)

\begin{abstract}
We numerically investigate by the finite-difference time-domain method the effects of surface termination on directional emission exiting a photonic crystal waveguide. The directed power and far-field beam profile for the original proposal [E. Moreno et al., Phys. Rev. B 69, 121402 (2004)] and its enhancement [S. K. Morrison et al., Appl. Phys. Lett. 86, 081110 (2005)] are computed for different values of some important parameters. We find another surface termination condition with a positive surface displacement in the structure of the original proposal which has a negative surface displacement. Our surface termination is more effective than the original structure, and nearly as effective as the termination for the enhancement, for directional emission. Besides, our termination is simpler than that for the enhancement. We confirm the effectiveness of directional emission from our termination in its far-field beam profile, radiation intensity distribution, and additionally the wave-vector space representation by the Fourier analysis.
\end{abstract}

OCIS codes : $050.0050,240.6690$

\section{INTRODUCTION}

Several years ago, beaming phenomenon from a single subwavelength aperture surrounded by a periodic corrugation on a metallic film was reported [1,2]. This phenomenon surprisingly overcomes the well-known diffraction limit that light emitting from an aperture much smaller than its wavelength spreads in all directions uniformly. The beaming originates from the excitation of surface plasmons on metallic surfaces, which act as parallel light sources due to the periodic corrugation. By applying the basic concept used for metallic films, beaming or directional emission exiting from a photonic crystal (PC) waveguide less than a wavelength wide was theoretically [3] and experimentally [4] demonstrated. The two pioneering reports stimulated a number of subsequent papers [5-10]. Surface waves on PC surfaces are intentionally generated by surface modification or termination, in contrast to surface plasmons occurring naturally on metallic surfaces.

Recently, directional emission from PCs with no surface waves involved has been reported in many publications [11-17]. Refs. [11] and [12] attain directional emission and beam deflection via defect coupling by defects placed just near the exit of a PC waveguide. In Ref. [13], a PC waveguide exit surface is covered by another different PC structure with self-collimation property that causes beaming emission. In Ref. [14], waveguiding inside a PC relies on self-collimation and surface modification makes directional emitters or beam splitters possible. Another proposed way is to put coupledresonator optical waveguides at the output surface of a PC waveguide [15]. Multiple adjacent waveguides can be used to obtain directional beam from light originating from a waveguide [16] or self-collimation [17]. The methods not relying on surface waves have some advantages, for example, simple surface structures, but they have limited controllability in the structures $[11$, 12] or complexity in the overall structures [13,15-17]. We believe that the methods for directional emission via surface waves have more flexibility and controllability. Beaming from PCs would find applications in nano-focusing and near-field optical devices. It may also be utilized in the coupling of PC waveguides with conventional photonic devices such as dielectric waveguides and optical fibers.

The directional emission from two-dimensional PC waveguides proposed originally in Ref. [3] was later enhanced by engineering the surface and adjusting relevant parameters $[7,8]$. These modifications include using a positive surface corrugation, increasing the refractive index of surface cylinders, inducing a near-surface defect 
mode, and adjusting the input wavelength in consequence [7]. We note here that the structure considered in Ref. [3] was not optimized. While a significant enhancement was achieved in Ref. [7], their procedure for optimization seems difficult to follow in terms of the underlying physics. Besides, the resulting surface structure is rather complicated for actual realization. In this paper, we numerically investigate the effects of surface termination on directional emission by the finite-difference time-domain method [18-20], mostly following the conditions of Refs. [3] and [7]. While computing directed power and far-field beam profiles for different values of some important parameters, we find another surface termination condition with a positive surface displacement in the structure of the original proposal which has a negative surface displacement. Our surface termination is more effective than the original structure, and nearly as effective as the termination for the enhancement, for directional emission. Besides, our termination is simpler than the one for the enhancement. We confirm the effectiveness of directional emission from our termination in its far-field beam profile and radiation intensity distribution, and additionally in the wave-vector space representation by the Fourier analysis [21-23].

\section{MODEL AND METHODS OF COMPUTATION}

The photonic crystal structure that we study here is shown in Fig. 1. Its basic structure is adopted from Refs. [3] and [7]. The underlying PC is a square lattice of dielectric cylinders in air $\left(\varepsilon_{r}=11.56\right.$ and $r=0.18 a$, where $r$ is the radius of cylinders and $a$ is the lattice constant.). We consider TM polarization (the electric field parallel to the axis of cylinders), for which the PC has a band gap from $\omega a / 2 \pi c=0.30$ to $\omega a / 2 \pi c=0.44$ ( $c$ is the speed of light.). At the center of the structure, a waveguide is formed by eliminating a line of cylinders. The radii of the surface cylinders at the PC-air interface are reduced to $r_{\mathrm{s}}=0.09 a$ to induce surface modes [5], which are nonradiative modes localized at the interface. In Fig. 1, $r_{s-1}$ is the radius of the cylinders next to the surface cylinders. To obtain directional

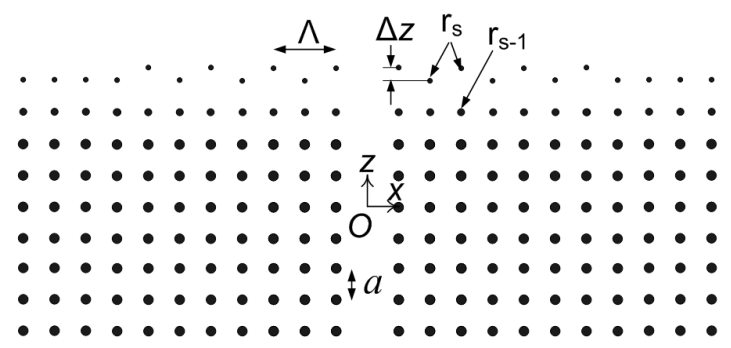

FIG. 1. Schematic of the photonic crystal structure for the computation of directional emission. emission by a coherent action of radiative surface modes [10], surface corrugation with displacement $\Delta z$ and period $\Lambda$ is created at the interface. This corrugation moves surface mode momentum, by adding its corrugation momentum, into the light cone [5], in which propagating modes in air reside in a dispersion diagram. Emission by radiative surface modes could also be understood by considering that the displaced cylinders by corrugation act as scattering centers for surface modes. Thus the surface corrugation displacement is an important parameter that determines the extent of surface emission by surface modes.

In its original proposal, Ref. [3] considered the case with a termination of $r_{\mathrm{s}}=0.09 a, n_{\mathrm{s}}=n=\sqrt{\epsilon_{r}}=3.4$ ( $n_{\mathrm{s}}$ is the refractive index of surface cylinders), and $r_{\mathrm{s}-1}$ $=r=0.18 a$, and with a source frequency of $\omega a / 2 \pi c$ $=0.408$. To make surface corrugation for inducing radiative surface modes, the even-numbered surface cylinders (counted according to their distance to the waveguide) were displaced with displacement $\Delta z=$ $-0.3 a$ and period $\Lambda=2 a$. The number of the displaced cylinders, $N$, was varied. In the structure for enhancement, Ref. [7] changed the termination to one with $r_{\mathrm{s}}$ $=0.09 a, n_{\mathrm{s}}=3.6$, and $r_{\mathrm{s}-1}=0.135 a$, and with a source frequency of $\omega a / 2 \pi c=0.379$. Besides, the odd-numbered surface cylinders with fixed $N=9$ were displaced with displacement $\Delta z=+0.4 a$ and period $\Lambda=2 a$.

To compute directional emission and relevant properties, we use the finite-difference time-domain (FDTD) method $[18,20]$ with a perfectly-matched-layer absorbing boundary condition [19]. Its basic algorithm that solves the Maxwell's equations directly in space and time is well-known and documented in details in Refs. [18,20]. We place a single-frequency sinusoidal source at the entrance of the waveguide $(x=0$ and $z=-4 a)$, to excite waves that propagate along the waveguide and are then emitted at its exit. To characterize the emission from the waveguide, we measure the time-averaged directed power $P_{\mathrm{d}}$ incident upon a cross section of width $2 a$ centered at $x=0$ and $z=41 a$, approximately $37 a$ away from the surface. Furthermore, the far-field beam profile of the directional emission is measured along the line of width $40 a$, perpendicular to the $z$ axis, centered at $x=0$ and $z=41 a$.

For the purpose of comparing different emissions more clearly, we adopt the Fourier analysis [21] in which the field distribution obtained from the FDTD method is converted into the wave vector space. The Fourier analysis was originally proposed to analyze reflection and refraction at an interface between a homogeneous medium and a PC [21], and later used to analyze a PC gradient index lens [22] or self-collimated beams in PCs [23]. In our work, the wave-vector space representation shows the shapes or profiles of wave-vector components excited after exiting a waveguide, thereby enabling one to compare spatial patterns of different directional beams. 


\section{RESULTS AND DISCUSSION}

In Fig. 2, we show the effect of surface displacement $\Delta z$ on directional emission. The surface displacement is an important parameter, because it makes a surface grating and causes nonradiative surface modes to become radiative by adding its corrugation momentum, as described previously. Figure 2 (a) shows the directed power $P_{\mathrm{d}}$ for different values of the surface displacement $\Delta z$ for the surface conditions of Refs. [3] and [7]. In Ref. [3], the authors used a negative surface corrugation of $\Delta z=-0.3 a$ for its surface grating, at which reasonably high $P_{\mathrm{d}}$ is obtained as shown in Fig. 2 (a). In contrast, Ref. [7] used a positive surface corrugation of $\Delta z=+0.4 a$ to obtain its enhanced emission. Its value of the surface corrugation at which maximum $P_{\mathrm{d}}$ is obtained is corroborated in Fig. 2 (a). As expected, the directed power $P_{\mathrm{d}}$ varies significantly for different values of $\Delta z$, which confirms that the amount of surface displacement determines the extent of emission.

For the plot of Ref. [3] in Fig. 2 (a), we notice another value of $\Delta z=+0.5 a$ that gives large directed power, although it is still smaller than that for Ref. [7]. So, in Fig. 2 (b) we plot the far-field beam profiles at $z$ $=41 a$, normalized to each maximum value, for the three cases. We easily see that the case for Ref. [7] with $\Delta z$ $=+0.4 a$ and one for Ref. [3] with $\Delta z=+0.5 a$ give well-collimated beams, whereas the case for Ref. [3] with $\Delta z=-0.3 a$ gives a beam collimated somewhat with large sidelobes. From these results, we see that a simple change of surface displacement gives significantly different properties of the far-field beam profiles. For further analysis, we call the condition of Ref. [7] with $\Delta z=$ $+0.4 a$ as Termination A, that of Ref. [3] with $\Delta z=$ $+0.5 a$ as Termination $\mathrm{B}$, and that of Ref. [3] with $\Delta z$
$=-0.3 a$ as Termination $\mathrm{C}$.

Figure 3 shows the effect of the number of displaced surface cylinders, $N$, for the three termination conditions just mentioned. In Fig. 3 (a), the directed power for all three terminations increases up to certain values of $N$. This is as expected because gratings for radiative surface modes must be formed to obtain directional emission. For Termination A, minimum $N$ $=8$ is needed for maximum $P_{\mathrm{d}}$, while for Termination $\mathrm{B}$, minimum $N=6$ is needed. However, for Termination $\mathrm{C}, P_{\mathrm{d}}$ reaches a maximum around $N=4$, and then decreases and fluctuates. This is due to variation of the beam profiles, which will be shown below. Figures 3 (b) and 3 (c) give the far-field beam profiles for different values of $N$ for Termination A and B, respectively. The main beams of those profiles do not vary, accompanied by a little variation of the sidelobes. However, in Fig. 3 (d) for Termination C, we easily see a significant variation of the main beams as well as the sidelobes for different values of $N$. The asymmetry of the sidelobes in Fig. 3 (b) and of the main beam for $N=7$ in Fig. 3 (d) is believed to originate from numerical artifacts.

As noted previously, Ref. [7] enhanced its directional emission by using the increased refractive index of surface cylinders and a near-surface defect mode induced by reduced radii of near-surface cylinders, in addition to its positive surface corrugation and its adjusted input wavelength. Figure 4 shows the effects of variation of the refractive index of surface cylinders, $n_{\mathrm{s}}$, and of the radius of near-surface cylinders, $r_{\mathrm{s}-1}$, on the directed power $P_{\mathrm{d}}$ for Termination A. In Fig. 4 (a), we see that $P_{\mathrm{d}}$ is rather sensitive to $n_{\mathrm{s}}$ and has a maximum value at $n_{\mathrm{s}}=3.6$, different from $n=3.4$ of the underlying PC cylinders. In Fig. 4 (b), $P_{\mathrm{d}}$ is highly sensitive

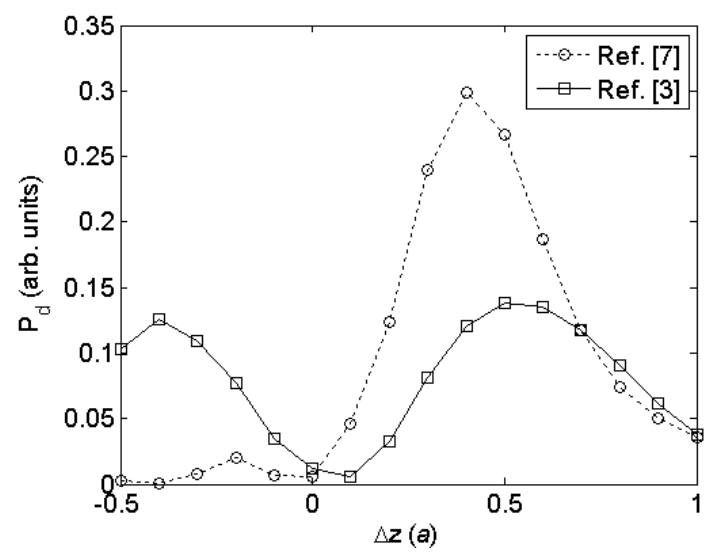

(a)

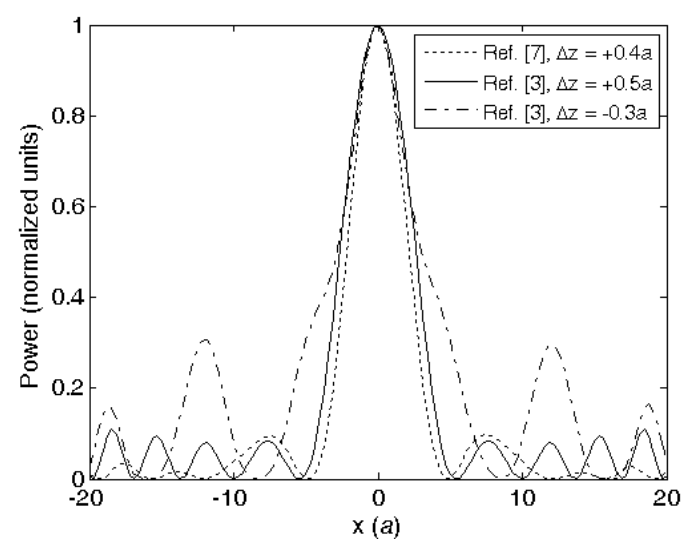

(b)

FIG. 2. For Ref. [3] in this Figure, the even-numbered surface cylinders with $N=9$ are displaced. For Ref. [7] in this Figure, the odd-numbered surface cylinders with $N=9$ are displaced. (a) Directed power $P_{\mathrm{d}}$ versus surface cylinder displacement $\Delta z$. (b) Power incident upon the cross section at $z=41 a$, normalized to each maximum value. We call the condition of Ref. [7] with $\Delta z=+0.4 a$ as Termination A, that of Ref. [3] with $\Delta z=+0.5 a$ as Termination B, and that of Ref. [3] with $\Delta z=-0.3 a$ as Termination C. 


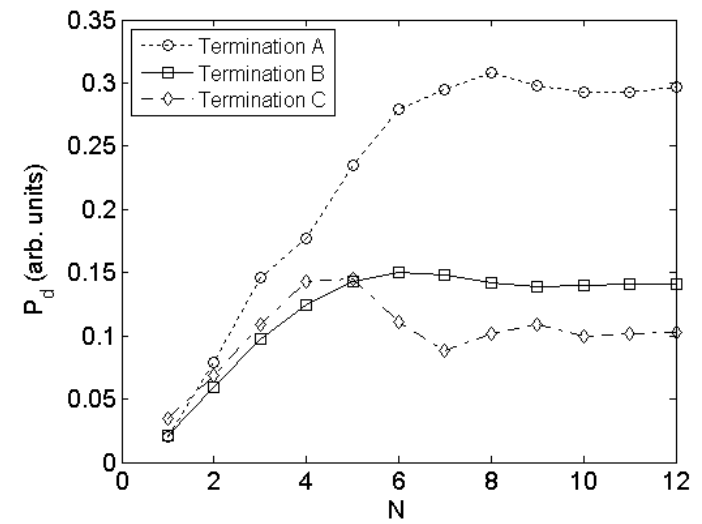

(a)

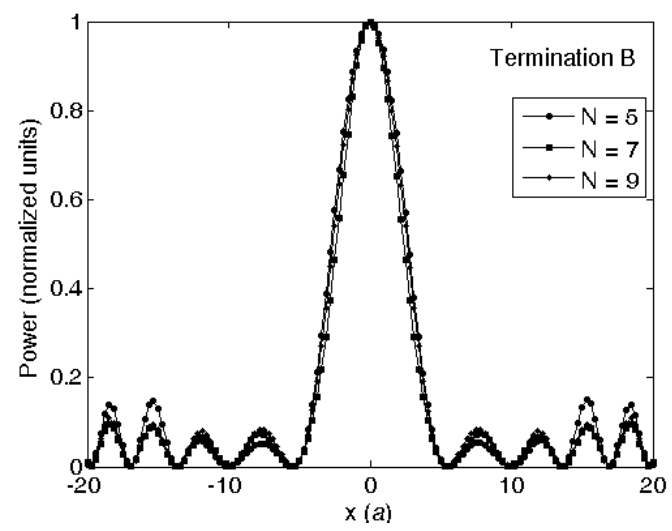

(c)

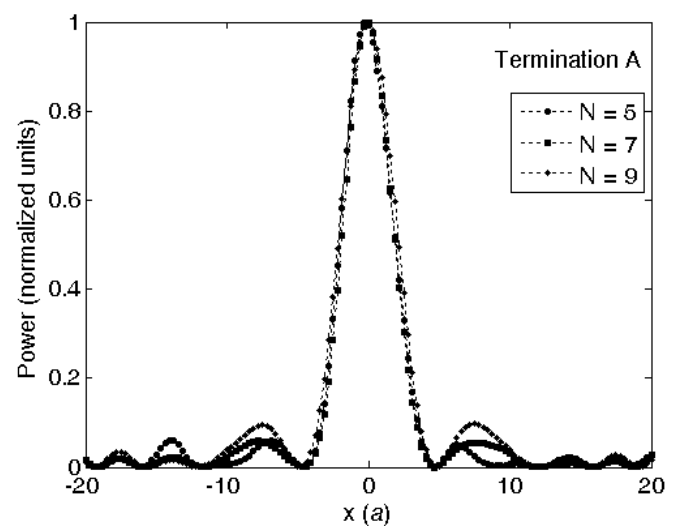

(b)

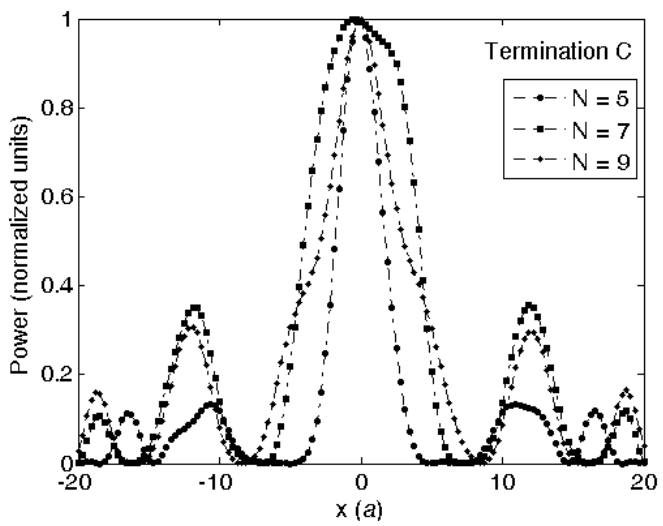

(d)

FIG. 3. (a) Directed power $P_{\mathrm{d}}$ versus the number of displaced surface cylinders, $N$; (b), (c), and (d) Power incident upon the cross section at $z=41 a$, normalized to each maximum value, for Termination A, B, and C, respectively.

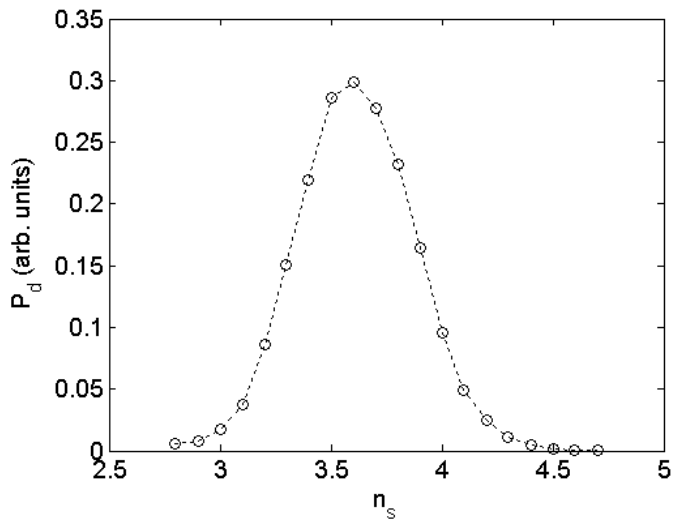

(a)

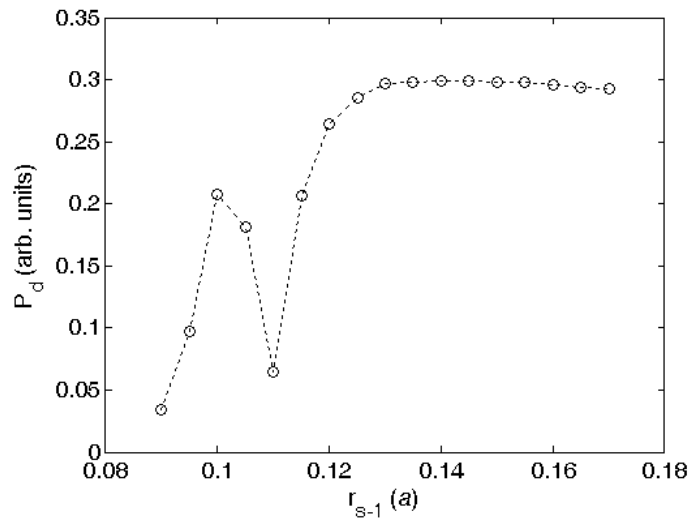

(b)

FIG. 4. Directed power $P_{\mathrm{d}}$ versus (a) the refractive index of surface cylinders, $n_{\mathrm{s}}$, with $r_{\mathrm{s}-1}=0.135 a$ and (b) the radii of near-surface cylinders, $r_{\mathrm{s}-1}$, with $n_{\mathrm{s}}=3.6$, both for Termination A.

to $r_{\mathrm{s}-1}$ up to a certain value of $r_{\mathrm{s}-1}=0.135 a$ at which maximum $P_{\mathrm{d}}$ is obtained. However, the deviation of $r_{\mathrm{s}-1}$ from $r=0.18 a$ of the PC cylinders does not seem to enhance the power significantly. We note that $n_{\mathrm{s}}=3.6$ and $r_{\mathrm{s}-1}=0.135 \mathrm{a}$ are the optimal values used in Ref. [7] and that $P_{\mathrm{d}}$ is highly sensitive to these two para- meters. The abrupt variation of $P_{\mathrm{d}}$ around $r_{\mathrm{s}-1}=0.11 a$ in Fig. 4 (b) may be from the numerical inaccuracy originating from the staircase approximation used in resolving circular structures, even though the grid size of $a / 64$ used here is small enough for most cylinders.

Figure 5 presents the intensity distributions of the 


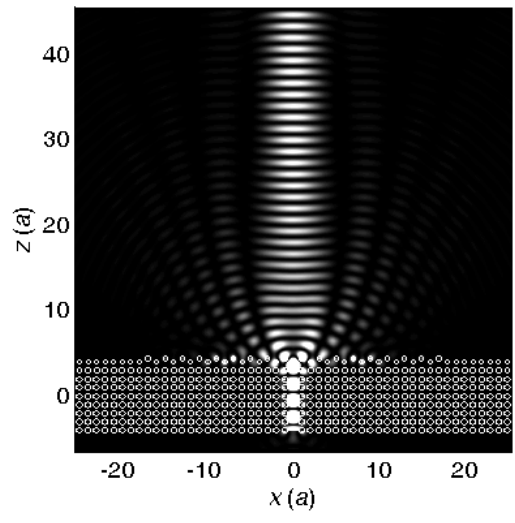

(a)

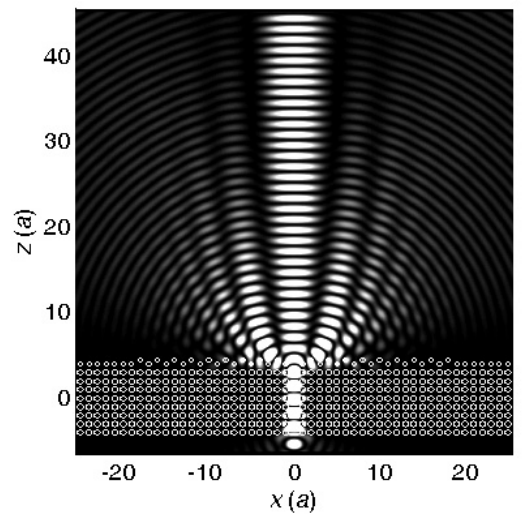

(b)

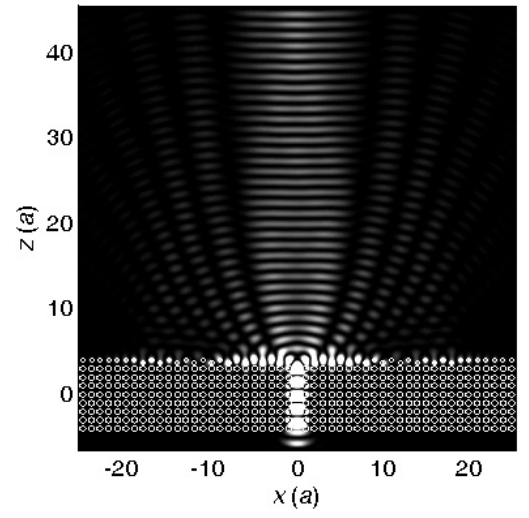

(c)

FIG. 5. Intensity distribution of directional emission for: (a) Termination A; (b) Termination B; (c) Termination C.

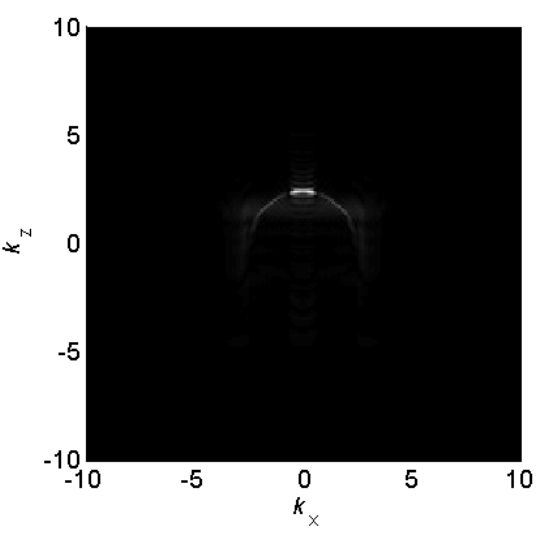

(a)

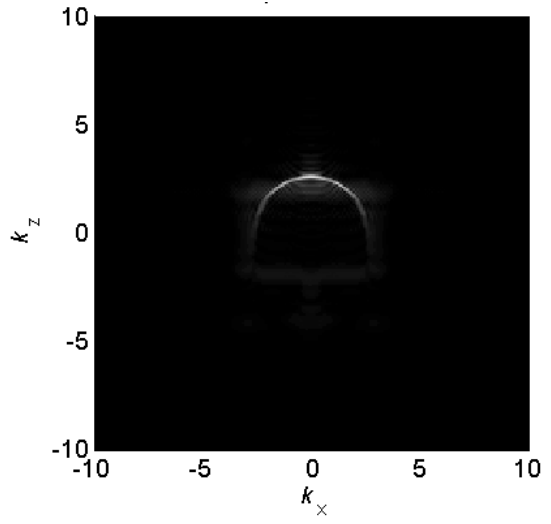

(b)

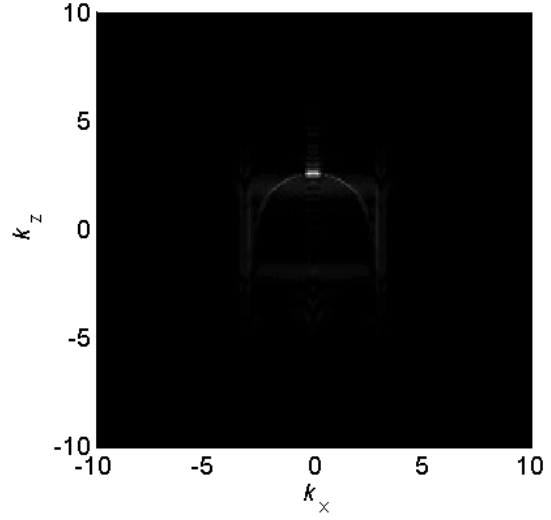

(c)

FIG. 6. Wave-vector space representation of directional emission by the Fourier analysis for: (a) Termination A; (b) Termination B; (c) Termination C.

directional emission from the three terminations. For Termination A in Fig. 5 (a), which is an optimum condition of Ref. [7], we see a well-collimated directional beam, almost identical to the result given in the reference. Termination B in Fig. 5 (b) is the condition that we have found in this work. This condition excites another well-collimated directional beam comparable to one in Fig. 5 (a). Termination C in Fig. 5 (c) is the condition described in Ref. [3]. This termination also excites a directional beam that seems to be collimated, but not as well as the previous two figures.

As described above, the wave-vector space representation enables one to compare spatial patterns of different directional beams. Figure 6 gives the results by the Fourier analysis in the wave-vector space for the three terminations. The equifrequency contour present in the wave-vector space is a locus of the wave vectors of possible propagating waves [21]. It is a circle in a two-dimensional, homogeneous, and isotropic medium such as air. Bright areas on the contour are the components of the wave vectors that are excited by actual propagating waves. Figures 6 (a) - 6 (c) show excited wave vector components for Termination A, B, and C, respectively. We note here that the width of an excited wave vector is inversely proportional to the width of a beam in real space. Comparing Figs. 6 (a) and 6 (b), we see that the widths of the main bright areas at the center are almost comparable, although Fig. 6 (b) has other bright components away from the center. This fact is consistent with the similarity of the intensity distributions in Figs. 5 (a) and 5 (b). Figure 6 (c) shows the much narrower width of the excited wave vector, which is again consistent with the wider directional beam shown in Fig. 5 (c). Figure 6 might be somewhat difficult to recognize visually due to the black-and-white printing. So the reader is advised to see many color figures for the equifrequency contours of air in Ref. [21] and to compare them to Figure 6 .

\section{CONCLUSION}

In this paper, we have numerically investigated by the finite-difference time-domain method the effects of 
surface termination on directional emission exiting a photonic crystal waveguide. The directed power and far-field beam profile for the conditions of the original report and the subsequent report on its enhancement have been computed for different values of some important parameters. We have found another surface termination condition with a positive surface displacement in the structure of the original proposal which has a negative surface displacement. Our surface termination is more effective than the original structure, and nearly as effective as the termination for the enhancement, for directional emission. Our termination does not require the increased index of surface cylinders, decreased radii of near-surface cylinders, and source frequency adjustment. So our termination is simpler than the one for the enhancement. We have confirmed the effectiveness of directional emission from our termination in its far-field beam profile, radiation intensity distribution, and additionally the wave-vector space representation by the Fourier analysis.

\section{ACKNOWLEDGEMENT}

This work was supported by the 2008 Hongik University Research Fund.

*Corresponding author: kbchung@hongik.ac.kr

\section{REFERENCES}

[1] H. J. Lezec, A. Degiron, E. Devaux, R. A. Linke, L. Martin-Moreno, F. J. Garcia-Vidal, and T. W. Ebbesen, "Beaming light from a subwavelength aperture," Science, vol. 297, no. 5582, pp. 820-822, 2002.

[2] L. Martin-Moreno, F. J. Garcia-Vidal, H. J. Lezec, A. Degiron, and T. W. Ebbesen, "Theory of highly directional emission from a single subwavelength aperture surrounded by surface corrugations," Phys. Rev. Lett., vol. 90, no. 16, pp. 167401-1167401-4, 2003.

[3] E. Moreno, F. J. Garcia-Vidal, and L. Martin-Moreno, "Enhanced transmission and beaming of light via photonic crystal surface modes," Phys. Rev. B, vol. 69, no. 12, pp. 121402-1-121402-4, 2004.

[4] P. Kramper, M. Agio, C. M. Soukoulis, A. Birner, F. Müller, R. B. Wehrspohn, U. Gösele, and V. Sandoghdar, "Highly directional emission from photonic crystal waveguides of subwavelength width," Phys. Rev. Lett., vol. 92, no. 11, pp. 113903-1-113903-4, 2004.

[5] E. Moreno, L. Martin-Moreno, and F. J. Garcia-Vidal, "Efficient coupling of light into and out of a photonic crystal waveguide via surface modes," Photonics Nanostruc. - Fundamen. Appl., vol. 2, no. 2, pp. 97-102, 2004.

[6] I. Bulu, H. Caglayan, and E. Ozbay, "Beaming of light and enhanced transmission via surface modes of photonic crystals," Opt. Lett., vol. 30, no. 22, pp. 30783080, 2005.

[7] S. K. Morrison and Y. S. Kivshar, "Engineering of direc- tional emission form photonic-crystal waveguides," Appl. Phys. Lett., vol. 86, no. 8, pp. 081110-1-081110-3, 2005.

[8] S. K. Morrison and Y. S. Kivshar, "Beaming effect and directional emission from photonic-crystal waveguides," Proc. SPIE, vol. 5733, pp. 104-113, 2005.

[9] W. Smigaj, "Model of light collimation by photonic crystal surface modes," Phys. Rev. B, vol. 75, no. 20, pp. 205430-1-205430-8, 2007.

[10] K. B. Chung, "Properties of surface modes used for directional emission from photonic crystal waveguides," J. Opt. Soc. Korea, in press, 2008.

[11] C.-C. Chen, T. Pertcsh, R. Iliew, F. Lederer, and A. Tünnermann, "Directional emission from photonic crystal waveguides," Opt. Express, vol. 14, no. 6, pp. 24232428, 2006.

[12] K. Guven and E. Ozbay, "Directivity enhancement and deflection of the beam emitted from a photonic crystal waveguide via defect coupling," Opt. Express, vol. 15, no. 23, pp. 14973-14978, 2007.

[13] D. Tang, L. Chen, and W. Ding, "Efficient beaming from photonic crystal waveguides via self-collimation effect," Appl. Phys. Lett., vol. 89, no. 13, pp. 131120-1131120-3, 2006.

[14] W. Y. Liang, J. W. Dong, and H. Z. Wang, "Directional emitter and beam splitter based on self-collimation effect," Opt. Express, vol. 15, no. 3, pp. 1234-1239, 2007.

[15] Z.-H. Zhu, W.-M. Ye, J.-R. Ji, X.-D. Yuan, and C. Zen, "Enhanced transmission and directional emission via coupled-resonator optical waveguides," Appl. Phys. $B$, vol. 86, pp. 327-331, 2007.

[16] Z. Li, K. Aydin, and E. Ozbay, "Highly directional emission from photonic crystals with a wide bandwidth," Appl. Phys. Lett., vol. 91, no. 12, pp. 121105-1-121105-3, 2007.

[17] Y. Zhang, Y. Zhang, and B. Li, "Highly-efficient directional emission from photonic crystal waveguides for coupling of freely propagated terahertz waves into $\mathrm{Si}$ slab waveguides," Opt. Express, vol. 15, no. 15, pp. 92819286, 2007.

[18] K. S. Yee, "Numerical solution of initial boundary value problems involving Maxwell's equations in isotropic media," IEEE Trans. Antennas Propag., vol. AP-14, no. 3, pp. 32-307, 1966.

[19] J. P. Berenger, "A perfectly matched layer for the absorption of electromagnetic waves," J. Comput. Phys., vol. 114, no. 2, pp. 185-200, 1994.

[20] A. Taflove and S. C. Hagness, Computational Electrodynamics: The Finite-Difference Time-Domain Method (Third Edition, Artech House, Boston, U.S.A., 2005).

[21] I. D. Leon and F. S. Roux, "Fourier analysis of reflection and refraction in two-dimensional photonic crystals," Phys. Rev. B., vol. 71, no. 23, pp. 235105-1-235105-10, 2005.

[22] F. S. Roux and I. D. Leon, "Planar photonic crystal gradient index lens, simulated with a finite difference time domain method," Phys. Rev. B., vol. 74, no. 11, pp. 113103-1-113103-4, 2006.

[23] A. Kim, K. B. Chung, and J.W.Wu, "Control of selfcollimated Bloch waves by partially flat equifrequency contours in photonic crystals," Appl. Phys. Lett., vol. 89, no. 25, pp. 251120-1-251120-3, 2006. 